\title{
Electroluminescence at Si Bandgap Energy from Metal-Oxide-Semiconductor Tunneling Diodes
}

\author{
Ching-Fuh Lin*, Miin-Jang Chen*, Ming-Hung Lee, and Cheewee Liu \\ Department of Electrical Engineering, National Taiwan University, Taipei 106, Taiwan \\ Email: cflin@cc.ee.ntu.edu.tw \\ * also with Graduate Institute of Electro-Optical Engineering
}

\begin{abstract}
We report room-temperature electroluninescence at Si bandgap energy from Metal-Oxide-Semiconductor (MOS) tunneling diodes. The ultrathin gate oxide with thickness $1 \sim 3 \mathrm{~nm}$ was grown by rapid thermal oxidation (RTO) to allow significant current to tunnel through. The measured EL efficiency of the MOS tunneling diodes increases with the injection current and could be in the order of $10^{-5}$, which exceeds the limitation imposed byindirect bandgap nature of $\mathrm{Si}$. We also study the temperature dependence of the electroluminescence and photoluminescence. The electroluminescence is much less dependent on temperature than photoluminescence from Si. The applied external field that results in the accumulation of majority carriers at $\mathrm{Si} / \mathrm{SiO}_{2}$ interface in the case of electroluminescence could be the reason for such difference. The involved physics such as optical phonon, interface roughness, localized carriers, and exciton radiative recombination are used to explain the electroluminescence from silicon MOS tunneling diodes .
\end{abstract}

Keywards - Silicon, MOS, electroluminescence.

\section{Introduction}

Silicon is the most important semiconductor material for electronics industry, but has limited applications in area of optics and optoelectronics due to its indirect bandgap characteristics that make it hardly emit light. In order to convert silicon to a light-emitting material, many efforts had been tried before, including porous silicon, nanocrystalline $\mathrm{Si}, \mathrm{Si} / \mathrm{Ge}$ alloy, and Erbium doping of $\mathrm{Si}$, and so on '. However, this luminescence originates from the defect-related mechanisms and the fabrication processes are complicated. In this paper, we report efficient electroluninescence (EL) at Si bandgap from metal/ $\mathrm{SiO}_{2} / \mathrm{Si}$ Metal-Oxide-Semiconductor tunneling diodes ${ }^{2,3}$. The characteristics of the light-emitting MOS tunneling diodes on silicon are

1. The structure of MOS tunneling diode is the same as that used in Ultra-Large-Scale-Integration (ULSI) circuits ,

2. Using tunneling current through the ultrathin oxide $(15 \sim 35 \AA)$ to provide carriers for radiative recombination,

3. The emission wavelength is near silicon bandgap, and

4. The fabrication processes are fully compatible with the present ULSI technology.

As a result, the light-emitting MOS tunneling diodes show promise to achieve the practical on-chip light source for optical interconnection and silicon-based optoelectronics integrated circuits (OEIC).

The temperature dependence of electro- and photoluminescence (PL) was also investigated to study the luminescent properties of the silicon MOS tunneling diodes. The EL was found to be less dependent on temperature than PL from Si. The reason is attributed to the accumulation of majority carriers at $\mathrm{Si} / \mathrm{SiO}_{2}$ interface due to the applied external field in the EL case. A physical process involving phonon assistance and exciton radiative recombination is used to fit the measured electroluminescence spectra. The excellent match of the calculated spectrum to the measured data of EL spectra shows that the exciton radiative recombination and the participation of optical phonon take place in the room-temperature EL from the silicon MOS tunneling diodes. 


\section{Device Fabrication}

When the devices are scaled down in the state-of-the-art ULSI chips, ultra-thin gate oxides are needed to reduce short channel effects. However, current tunneling through the ultra-thin gate oxide inevitably increases when the thickness of the gate oxide shrinks with each generation of IC fabrication technology. Therefore, the devices based on the tunneling current are fabricated and developed. In order to grow the high quality ultra-thin gate oxide, the rapid thermal oxidation (RTO) is commonly used to meet this requirement ${ }^{4}$.

In this work, the MOS tunneling diode has the ultrathin gate oxide to make significant current tunnel through. The ultrathin gate oxide was grown by rapid thermal oxidation (RTO) on n-type (100) Si wafer at $900-1000^{\circ} \mathrm{C}$. The resistivity of Si substrate are $1 \sim 10 \Omega-\mathrm{cm}$. The gas flows are $500 \mathrm{sccm}$ nitrogen and $500 \mathrm{sccm}$ oxygen at a reduced pressure. The thickness of oxide is measured by ellipsometry and confirmed by high-frequency capacitance-voltage measurements. The oxide thickness is in the range of $15 \sim 35 \AA$. Aluminum or transparent indium tin oxide (ITO) was deposited as the gate electrode using the usual evaporator. The gate electrodes with various circular areas are defined by photolithography. The thickness of Si substrate is about $500 \mu \mathrm{m}$ and aluminum was deposited on the backside as another electrode of the MOS diode. The device structures are schematically shown in Fig.1.

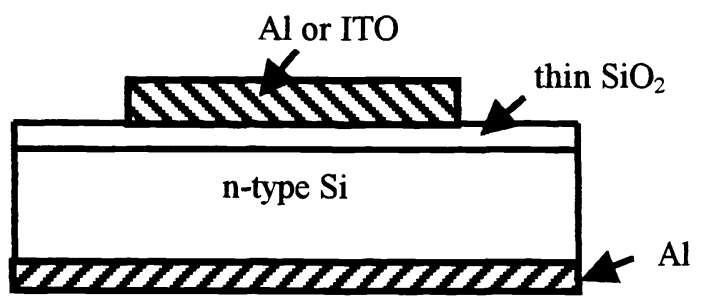

Fig. 1 schematic diagram of the MOS tunneling diode

\section{I-V Characteristics, Electroluminescence Spectra and Quantum Efficiency}

Fig. 2 shows the typical I-V characteristics of the MOS tunneling diodes on $\mathbf{n}$-type silicon and Fig. 3 is the schematic band diagram. The I-V curve shows the rectifying characteristics. When positive voltage is applied at the metal gate, the diode operates in the accumulation region. The positive gate voltage will inject holes from metal to silicon and attract electrons at the $\mathrm{Si} / \mathrm{SiO}_{2}$ interface to form an accumulation layer. The tunneling holes then recombine with the localized electrons in the accumulation layer to generate radiative emission, as schematically shown in Fig.3. The electrons accumulating at the $\mathrm{Si}_{/} \mathrm{SiO}_{2}$

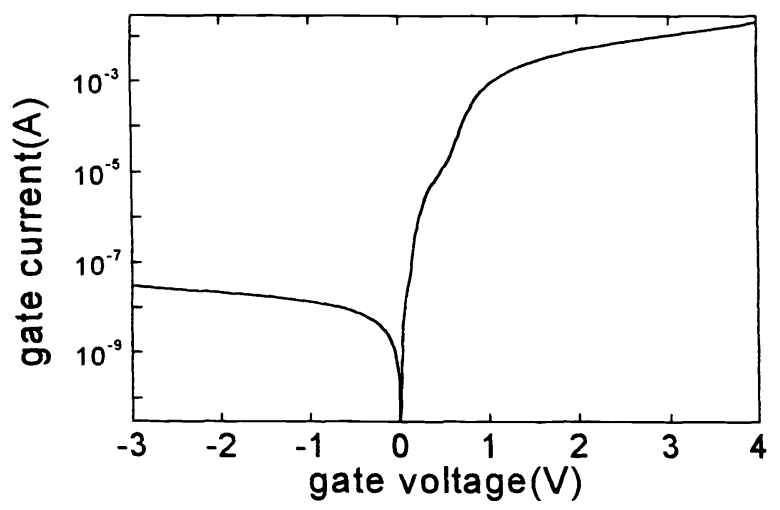

Fig.2 I-V characteristics of the tunneling diode on n-type Si substrate 
interface also tunnel through the thin oxide from the silicon to metal. The electron tunneling current is much larger than the hole tunneling current due to the larger tunneling barrier for hole. Even though the electron tunneling current acts as the leakage current for radiative recombination, EL was still observed. Note that there is a kink at about $0.8 \mathrm{~V}$, which implies two different current tunneling mechanisms ${ }^{5}$. At small positive gate voltage, the current was primarily contributed the electrons in conduction band tunneling from the silicon to metal gate through interface states. As the voltage increases above $0.8 \mathrm{~V}$, the holes tunneling from the metal to silicon contribute to the current. When the gate is biased at negative voltage, an inversion layer of the holes is formed at $\mathrm{Si} / \mathrm{SiO}_{2}$ interface. As long as the holes in the inversion layer are generated, they tunnel away to the metal gate and the inversion layer is always kept at the onset of the inversion region, instead of the stronger inversion. The reverse-biased current is limited by the generation rate of the holes through the $\mathrm{Si} / \mathrm{SiO}_{2}$ interface state and so is much smaller than the forward biased current.

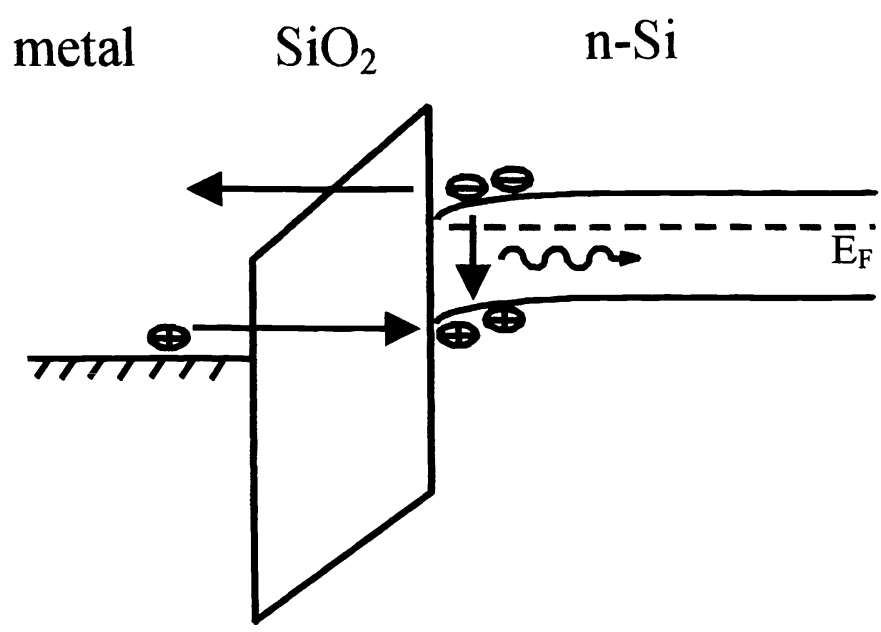

Fig. 3 Schematic band diagram of MOS tunneling diode operated in the accumulation region.

When significant current tunnels through the thin oxide, EL is clearly observed through an IR-viewer even with a very low injection current density, $<1 \mathrm{~A} / \mathrm{cm}^{2}$. The diode is operated at room temperature under continuous wave operation without cooling. Since the luminescence was almost blocked by the thick Al gate electrode, only the emission from the gate electrode edge can be measured. Fig. 4 shows the emission spectra at various injection current of $\mathrm{Al} / 30 \AA$ oxide/n-Si with a gate electrode area of $8 \times 10^{-3} \mathrm{~cm}^{2}$. These spectra were measured using an InGaAs detector placed at the exit of the monochromator. The emission wavelength is near the silicon bandgap energy.

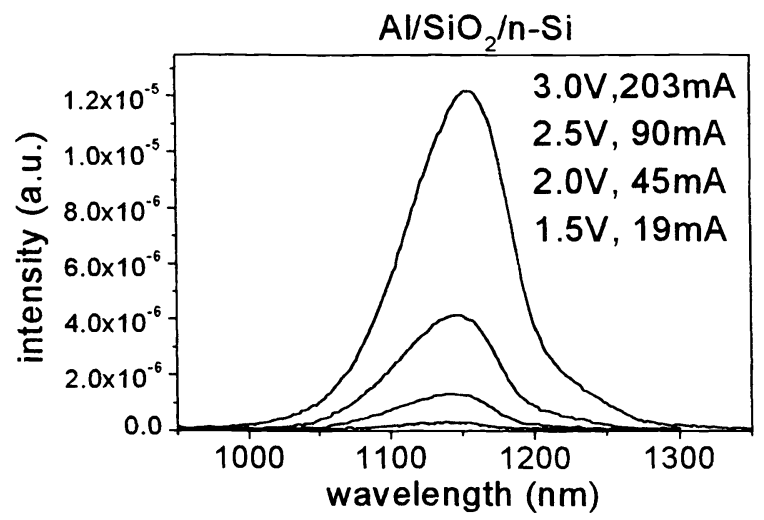

Fig.4 The electroluminescence spectra from $\mathrm{Al} / 30 \AA$ oxide/n-Si MOS tunneling diode 
Because the EL is almost blocked by the metal gate, we measure the optical power from the backside of the MOS diodes to estimate the quantum efficiency. Before the measurement, the $\mathrm{Al}$ metal gate at the back Si side was first lapped off. The backside Si surface was then polished mechanically and chemically. Afterward Al was redeposited, but not directly under the MOS area, as shown in Fig. 5. Hence the emitted power was not blocked by Al metal. Fig. 6 shows the measured spectrum from the back Si side and from the metal gate side. It demonstrates that the EL from the back Si side is narrower than that measured from the metal gate side because the short-wavelength part is absorbed by the Si substrate, which is about $500 \mu \mathrm{m}$ thick. Therefore, the measured optical power is attenuated by the absorption of the Si substrate. The measured L-I characteristics from the back Si side of the Al/30 $\AA$ oxide/n-Si MOS tunneling diode were shown in Fig,7. A superlinear increase of the output power with the injection current is shown. The efficiency increases with the injection current and the measured slope efficiency is $8 \times 10^{-6}$ at injection current $70 \mathrm{~mA}$. Note that the injection current density $\left(\sim 4 \mathrm{~A} / \mathrm{cm}^{2}\right)$ in this measurement is still
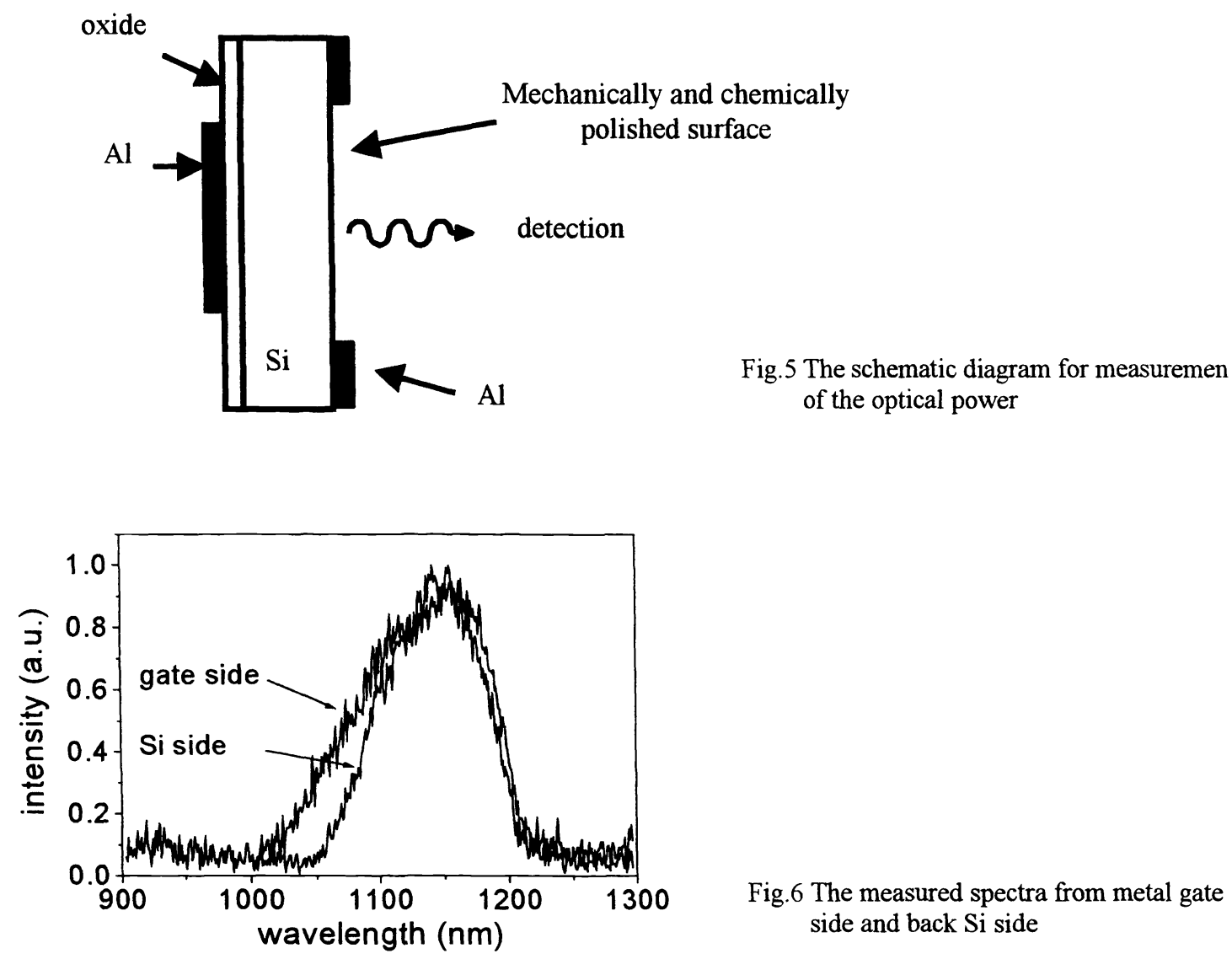

Fig.6 The measured spectra from metal gate side and back Si side

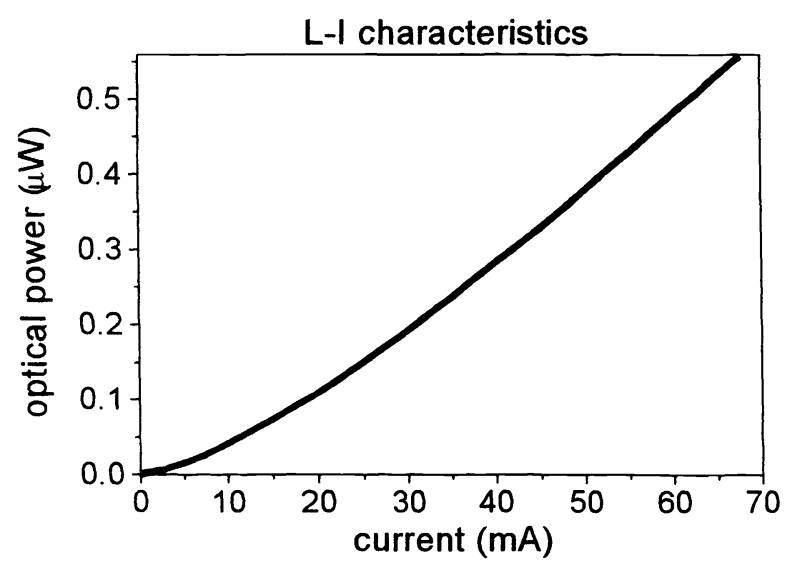

Fig.7 The measured L-I curves from the back Si side of the $A 1 / 30 \AA$ oxide/n-Si MOS tunneling diodes 
very low. The typical slope efficiency is estimated to be in the order of $10^{-5}$ for the room-temperature EL from MOS tunneling diodes on silicon. Better EL efficiency is expected with improved process condition and device structures, such as the current confinement and waveguide structures.

\section{Temperature Effects on Electroluminescence and Photoluminescence}

To further study the luminesent properties of the MOS tunneling diodes, the temperature dependence of electro- and photoluminescence was investigated ${ }^{6,7}$. The EL spectra of the Al/30 $\AA$ oxide/n-Si MOS diode from $300 \mathrm{~K}$ down to $98 \mathrm{~K}$ are shown in Fig.8. The gate voltage and injection current density are fixed at $3 \mathrm{~V}$ and $\sim 0.4 \mathrm{~A} / \mathrm{cm}^{2}$. Fig.9 shows the PL spectra from Si crystal under Argon laser excitation at different temperatures. Careful comparison between Fig. 8 and Fig. 9 reveals that the EL spectra are similar to PL ones. It is well known that the transverse optical (TO) phonon with energy $57.8 \mathrm{meV}$ is required for momentum conservation in PL. ${ }^{8}$ The resemblance between the electro- and photoluminescence spectra confirms the TO phonon participation of the TO phonon in the EL from MOS tunneling diodes on silicon.

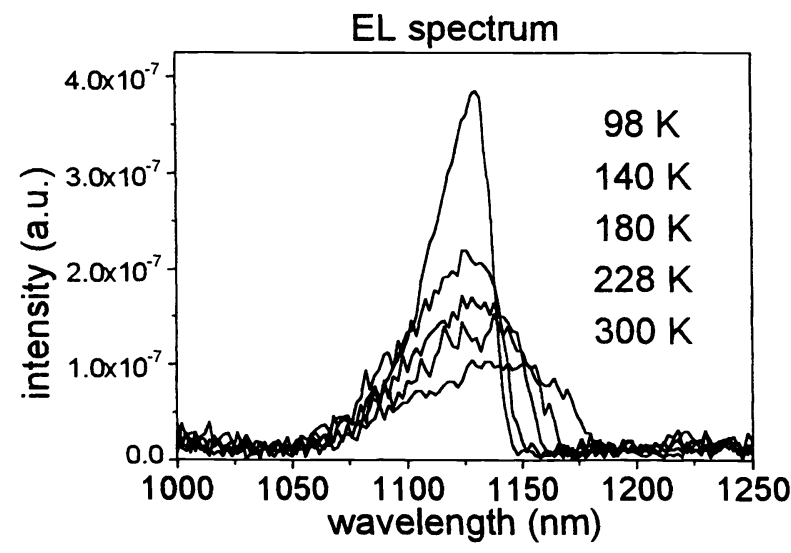

Fig. 8 The electroluminescence spectra from $\mathrm{Al} / 30 \AA$ oxide/n-Si MOS tunneling diod at various temperature

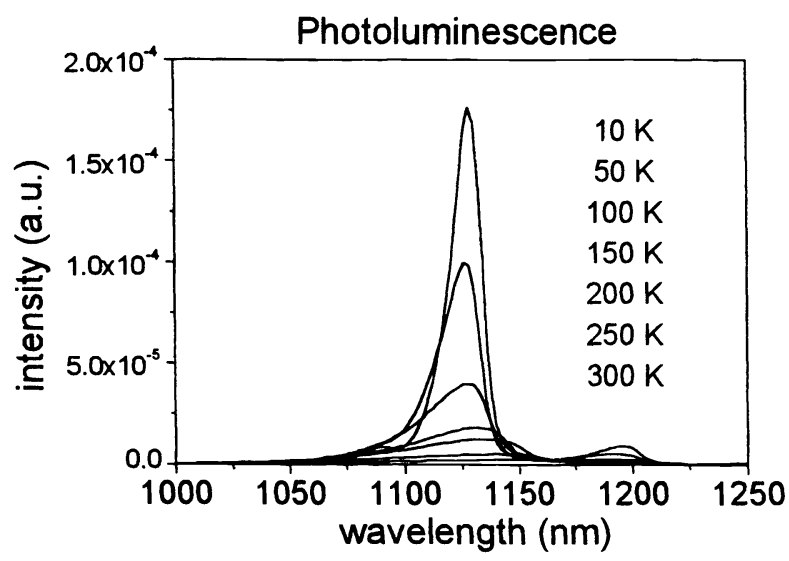

Fig.9 The photoluminescence spectra from Si crystal at various temperature

The EL was also discovered to be less dependent on temperature than the PL from Si. Fig. 10 shows the temperature dependence of electro- and photoluminescence intensity. The experiment demonstrates that the EL intensity of MOS 
tunneling diodes exhibits weak temperature dependence, as compared to PL. The reason is attributed to carrier confinement in the accumulation layer, which is related to the gate voltage and is almost independent of the temperature. On the other hand, the carrier confined in a small region is also captured by less impurity states, and therefore results in a reduced temperature dependence.

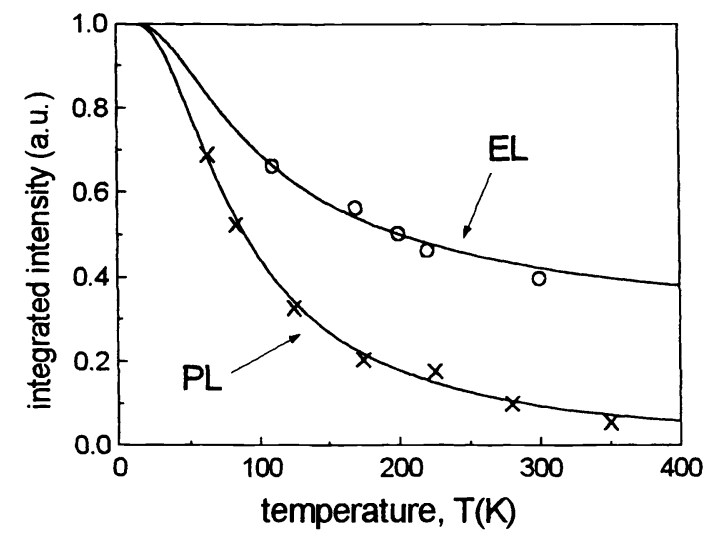

Fig.10 Temperature dependence of electroluminescence and phtotluminescence $(0, x$ : experimental results; solid line: theoretical prediction.)

\section{Radiative Recombination through Exciton}

We develop a physical model to describe the EL from the MOS tunneling on silicon. In order to fit the measured EL spectra, we found that the effect of exciton needs to be taken into account. Before recombination, an electron and a hole are first bounded together under Coulomb attraction to form an exciton, and then recombine to emit a photon and a phonon. ${ }^{9} \mathrm{We}$ derived the spectral shape of luminescence from $\mathrm{Si}$ in terms of the phonon-assisted and exciton radiative recombination as follows :

$$
\begin{aligned}
S(\hbar \omega) & \sum_{n} \frac{1}{(2 \pi)^{3}} \iint_{K q} d^{3} K d^{3} q \cdot\left|\phi_{n}(0)\right|^{2} L\left(E_{g}-\frac{E_{B}}{n^{2}}+\frac{\hbar^{2} K^{2}}{2 M}-E_{p}-\hbar \omega\right) \delta\left(\vec{q}-\vec{k}_{e}-\vec{k}_{h}\right) \frac{1}{\exp \left(\frac{E_{g}-\frac{E_{B}}{n^{2}}+\frac{\hbar^{2} K^{2}}{2 M}-F_{x}}{k_{B} T}\right)-1} \\
& +\frac{1}{(2 \pi)^{3}} \frac{1}{(2 \pi)^{3}} \iiint_{k K q} d^{3} k d^{3} K d^{3} q \cdot|\phi(0)|^{2} L\left(E_{g}+\frac{\hbar^{2} k^{2}}{2 \mu}+\frac{\hbar^{2} K^{2}}{2 M}-E_{p}-\hbar \omega\right) \delta\left(\vec{q}-\vec{k}_{e}+\vec{k}_{h}\right) \cdot \frac{1}{\exp \left(\frac{E_{g}+\frac{\hbar^{2} k^{2}}{2 \mu}+\frac{\hbar^{2} K^{2}}{2 M}-F_{x}}{k_{B} T}\right)-1}
\end{aligned}
$$

where $n$ is the quantum number of discrete exciton states; $K$ and $k$ are the center-of-mass and the relative wavevetor of exciton; $q$ is the wavevector of phonon; $k_{e}$ and $k_{h}$ are the wavevectors of the electron and hole composing the exciton, respectively; $M$ and $\mu$ are the total mass and the reduced mass of the electron-hole pair; $E_{g}$ is the temperature-dependent bandgap energy of $\mathrm{Si}$; $E_{B}$ is the exciton binding energy; $E_{P}$ is the energy of phonon participating the radiative transition; $F_{x}$ is the quasi-chemical potential of the exciton; $k_{B}$ is the Boltzmann constant and $T$ is the lattice temperature. The first term in the expression is the contribution from discrete exciton states. This term sums over all quantum numbers $n$. The second term corresponds to the ionized continuum exciton states. $\left|\phi_{n}(0)\right|^{2}$ and $|\phi(0)|^{2}$ are the probability of finding the electron and hole at the same space 
point. Because of the requirement of symmetric wavefunction in the spatial coordinate $\left(\left|\phi_{n}(0)\right|^{2}\right.$ and $\left.|\phi(0)|^{2} \neq 0\right)$, only the singlet spin state is taken into account in the expression. The Lorentzian linewidth broadening function $L$ is used to account for the effect of carrier scattering. The delta function in the integral represents the conservation of momentum. Because the phonon used to compensate the mismatch of momentum lies near the Brillouin zone edge, it is a good approximation to assume that the phonon energy $E_{P}$ is independent of its wavevector $q$. The temperature dependence of bandgap energy $E_{g}$ is given in Ref. 10. The widely accepted value of the exciton binding energy $E_{B}=14.7 \mathrm{meV}$ is used for the calculation. ${ }^{9}$ The emission of TO phonon with $E_{P}=57.8 \mathrm{meV}$ is known as the dominate mechanism for momentum conservation for radiative recombination. ${ }^{8}$ In the derivation of (1), we use the Bose-Einstein statistics obeyed by exciton. Assuming that the quasi-chemical potential $F_{x}$ of exciton is below the indirect bandgap over many $k T$, the Bose-Einstein function is reduced to the Boltzmann distribution. There are only two fitting parameters, the lattice temperature $T$ and the intra-band carrier scattering time $\tau_{s}$ in the Lorentzian function $L$, necessary to fit our theoretical lineshape (1) and the measured spectra from the MOS silicon tunneling diodes.

The EL spectra of the ITO/30 $\AA$ oxide/ $n$-Si tunneling diode are shown in Fig.11. The dashed lines are calculated spectra using (1). Because the measurement is performed at room temperature and the injection current density is low, $T=300 \mathrm{~K}$, $310 \mathrm{~K}$ and $\tau_{s}=60 \mathrm{fs}$ in the Lorentizian broadening function are used. The value of $\tau_{s}$ is in good agreement with the intraband carrier scattering time in Si. The long-wavelength tails in the Fig. 11 is due to the participation of two-phonon process in the radiative recombination. The PL spectnum of the same device at low temperature is shown in Fig. 12. There are three main peaks corresponding to two-phonon, TO, and TA phonon-assisted recombination processes. ${ }^{8}$ The two-phonon process involves the emission of a momentum-conserving TO phonon and a zone center $\left(\mathrm{O}^{\Gamma}\right)$ phonon. ${ }^{8}$ When the temperature increases to room temperature, the signal of two-phonon process in the PL spectrum gradually mixes with the main TO peak
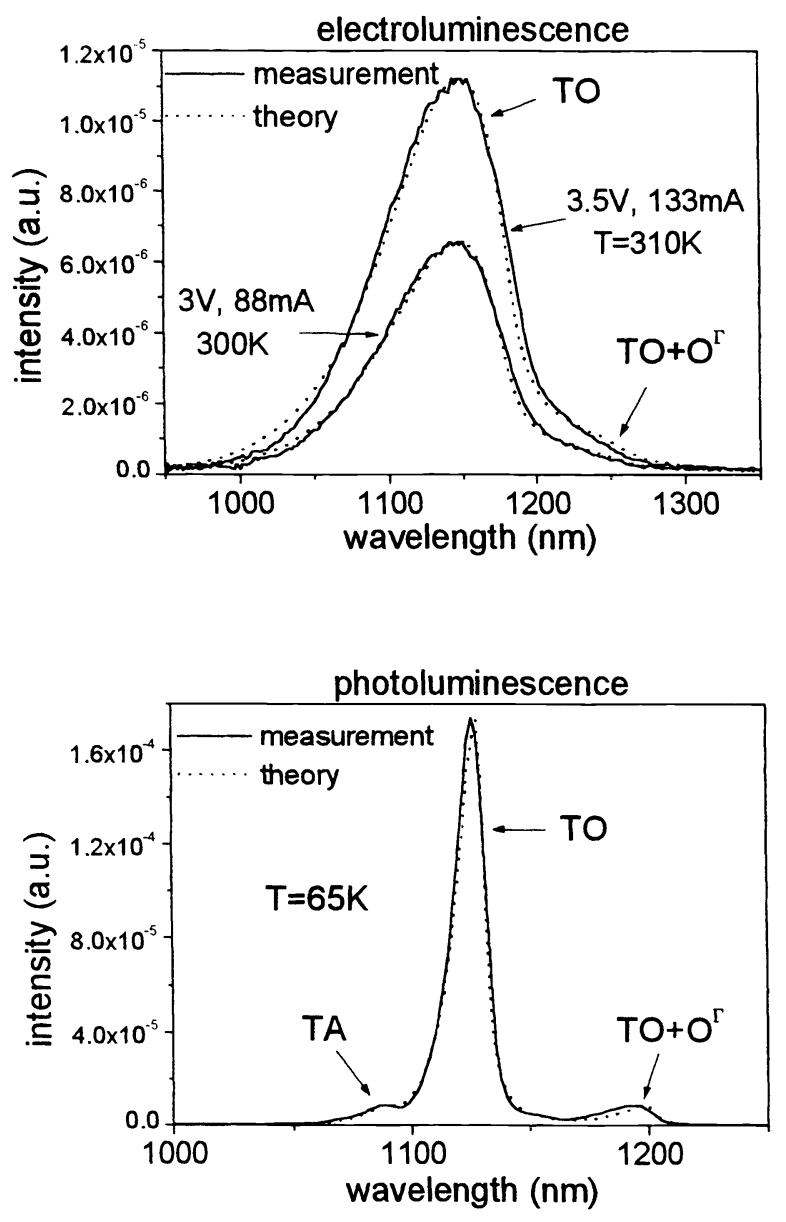

Fig.11 Room-temperature electroluminescenc spectra from $\mathrm{TTO} / 30 \AA \mathrm{SiO}_{2} / n$-Si tunnelin diode as well as the theoretical fitting.
Fig. 12 Low-temperature photoluminescence spectru from $\mathrm{TTO} / 30 \AA \mathrm{SiO}_{2} / n$-Si tunneling diode 
and forms the low-energy tail similar to Fig.11. The emission spectrum of two-phonon process is obtain using (1) with $E_{P}$ $=57.8+64.5 \mathrm{meV}\left(\mathrm{TO}+\mathrm{O}^{\Gamma}\right) .{ }^{8}$ The ratio of the magnitude between the two-phonon and TO peaks is 0.07 , which is the same as the relative magnitude of the two signals in the PL spectrum at $26 \mathrm{~K}{ }^{8}$ The excellent fitting of the theoretical spectrum to the measured data in Fig. 11 indicates that the exciton radiative recombination and the participation of optical phonon could take place in the room-temperature EL from the MOS silicon tunneling diodes.

\section{Discussions and Summary}

Possible reasons for the occurrence of EL from the MOS silicon tunneling diodes at room temperature are discussed as follows. Because of the indirect bandgap nature of $\mathrm{Si}$, some mechanisms are required for momentum conservation in the radiative recombination. In addition to the participation of phonons, the carrier scattering by $\mathrm{Si} / \mathrm{SiO}_{2}$ interface roughness is shown to be important to provide some momentum to compensate the momentum-mismatch between the electrons and holes in the k-space.

${ }^{11}$ Therefore, more phonons with momentum complementary to the interface roughness scattering are involved in the radiative recombination. The localized majority carriers in the accumulation region will also result in the spread of momentum in the $\mathrm{k}$-space and provide a similar function to that of the interface roughness. The spreading of wavefunction in the $\mathrm{k}$-space due to the interface roughness and localized carriers could enhance EL for Si.

It has been proposed that the effect of quantum confinement will assist the formation of excitons and enhance the probability of radiative transitions. ${ }^{12}$ The dimension of the quantum confinement system is typically in the order of the Bohr radius of exciton. The roughness at $\mathrm{Si} / \mathrm{SiO}_{2}$ boundary could provide the weak two-dimensional quantum confinement along the plane of interface. The carriers are also confined in the accumulation layer due to the applied electrical field. The interface roughness and electrical field then result in a three-dimensional quantum confinement for excitons. Therefore, in addition to the extra momentum contributed by the carrier scattering due to the interface roughness, the roughness could improve the formation of exciton for enhanced luminescence.

In summary, room-temperature EL from MOS tunneling diode on silicon was reported. The high-quality ultra-thin $\mathrm{SiO}_{2}$ layer was grown by the rapid thermal oxidation to allow significant tunneling current taking place. The emission wavelength corresponds to silicon bandgap energy and the slope efficiency couldbe in the order of $10^{-5}$. The temperature dependence of electro- and photoluminescence was also studied. The less temperature dependence of the electroluminescence is attributed to the carrier confinement in the accumulation layer. The resemblance between the electro- and photoluminescence spectra reveals the participation of the TO phonon in the EL. The EL for MOS silicon tunneling diodes is analyzed in terms of phonon-assisted and exciton radiative recombination. There is excellent match between the theoretical spectra and measured data. Some mechanisms, such as the carriers scattering by the $\mathrm{Si} / \mathrm{SiO}_{2}$ interface roughness, and the spread of momentum in $\mathrm{k}$-space due to the localized majority carriers in the accumulation layer, are used to explain the high-efficient radiative recombination in MOS silicon tunneling diodes.

\section{References}

1. David J. Lockwood, Light Emission in Silicon From Physics to Devices, Academic Press, 1998.

2. Ching-Fuh Lin, C. W. Liu, Miin-Jang Chen, M. H. Lee, and I. C. Lin, "Electroluminescence at Si Bandgap Energy Based on Metal-Oxide-Silicon Structures," J. Appl. Phys., 97, pp8793-8795, 2000.

3. C. W. Liu, M. H. Lee, Miin-Jang Chen, I. C. Lin, and Ching-Fuh Lin, "Room-Temperature electroluminescence from electron-hole plasmas in the metal oxide silicon tunneling diodes," Appl. Phys. Lett., 76, pp.1516-1518, 2000.

4. C.Y. Chang and S. M. Sze, ULSI technology, New York : McGraw-Hill, 1996.

5. A.Ghetti, et. al., Int. Electron Device Meeting, pp 731, 1999.

6. C. W. Liu, M-J Chen, I. C. Lin, M. H. Lee, and C-F Lin, "Temperature dependence of the electron-hole-plasma electroluminescence from the metal-oxide-silicon tunneling diodes," Appl. Phys, Lett. 77, pp.1111-1113, 2000.

7. Ching-Fuh Lin, Miin-Jang Chen, Eih-Zhe Liang, W.T. Liu, and C.W. Liu, "Reduced Temperature Dependence of Luminescence from Silicon due to Field-Induced Carrier Confinement," to be published in Appl. Phys. Lett., 2001

8. P. J. Dean, J. R. Haynes, and W. F. Flood, Phys. Rev. 161, 711 (1967).

9. R. A. Smith, Semiconductor, $2^{\text {nd }}$ ed, Cambridge University Press, New York, 1978.

10. V. Alex, S. Finkbeiner, "Temperature dependence of the indirect energy gap in crystalline silicon," J. Weber, J. Appl. Phys. 79, pp.6943-6946, 1996.

11. C. W. Liu, M. H. Lee, Miin-Jang Chen, and Ching-Fuh Lin, "Roughness-enhanced electroluminescence from metal-oxide-silicon tunneling diodes," IEEE Electron Device Lett. 21, pp,601-603, 2000.

12. P. F. Trwoga, A. J. Kenyon, and C. W. Pitt, "Modeling the Contribution of Quantum Confinement to Luminescence from Silicon Nanoclusters," J. Appl. Phys. 83, pp.3789-3794, 1998. 